\title{
Cytokeratin Expression Profiling in Gastric Carcinoma: Clinicopathologic Significance and Comparison with Tumor-Associated Molecules
}

\author{
Hokuto Takami Kazuhiro Sentani Miho Matsuda Naohide Oue \\ Naoya Sakamoto Wataru Yasui \\ Department of Molecular Pathology, Hiroshima University Graduate School of Biomedical Sciences, \\ Hiroshima, Japan
}

\section{Key Words}

Gastric cancer - Cytokeratin • Mucin phenotype •

Tumor-associated molecules

\begin{abstract}
Objective: The expressions of cytokeratin (CK) 7 and 20 have been studied in various primary and metastatic carcinomas, and their determination may help distinguish the site of origin of metastatic carcinomas. However, little is known about the molecular basis that determines variations in CK patterns in gastric cancers (GCs). The aim of the present study was to analyze the CK expression patterns in a large number of GCs and to investigate how the CK patterns correlate with clinicopathologic parameters, histology, mucin phenotype or several tumor-related molecules. Methods and Results: We immunohistochemically examined the CK7/CK20 patterns, mucin expression profiles (MUC5AC, MUC6, MUC2 and CD10), and the cancer-related molecules (CDX2, p53, EGFR and $\beta$-catenin), using a tissue microarray with $870 \mathrm{GCs}$. The GCs were divided into four patterns; $17 \%$ of CK7+/CK20+, 57\% of CK7+/CK20-, 9\% of CK7-/CK20+ and $17 \%$ of CK7-/CK20. GCs with the CK7-/CK20- pattern demonstrated a close relation to undifferentiated adenocarci-
\end{abstract}

noma. CK7 expression was significantly correlated with the expression of MUC5AC and MUC6, while CK20 expression was correlated with MUC2 and CDX2. There were statistically significant associations between CK expression patterns and mucin phenotypes. Conclusion: These results indicate that the CK7/CK20 expression patterns in GCs demonstrated different clinicopathologic features and molecular signatures.

Copyright $\odot 2012$ S. Karger AG, Basel

\section{Introduction}

Gastric cancer (GC) is one of the most common malignancies worldwide and often metastasizes to other organs, including the liver, lung and ovary [1]. In metastatic carcinomas of unknown primary site, identification of the origin as the stomach or other primary site is very difficult because of the heterogeneous histology of GC. This heterogeneity may be partly due to the fact that GC is caused by exogenous (nitrosamines and Helicobacter pylori) and endogenous (E-cadherin mutation) factors [2]. To identify these phenotypical differences at a morphologic level in a comparable and reproducible manner, it is

\section{KARGER}

Fax +4161306 1234

E-Mail karger@karger.ch

www.karger.com
() 2012 S. Karger AG, Basel

$1015-2008 / 12 / 0793-0154 \$ 38.00 / 0$

Accessible online at:

www.karger.com/pat
Wataru Yasui, $\mathrm{MD}, \mathrm{PhD}$

Department of Molecular Pathology

Hiroshima University Graduate School of Biomedical Sciences

1-2-3 Kasumi, Minami-ku, Hiroshima 734-8551 (Japan)

Tel. +81 82257 5145, E-Mail wyasui@ hiroshima-u.ac.jp 
necessary to have a novel classification system that recognizes all types of GCs adequately. In addition to classification by histology (the Lauren classification, the Japanese Classification of Gastric Carcinoma, etc.), GCs may also be classified into four phenotypes by the mucin expression profile: $\mathrm{G}$ type (gastric phenotype), I type (intestinal phenotype), GI type (gastric and intestinal mixed phenotype) and $\mathrm{N}$ type (neither gastric nor intestinal phenotype) $[2,3]$. The $\mathrm{G}$ type is considered to behave more aggressively than the I type. Mutations of $p 53$ and loss of heterozygosity of the adenomatous polyposis coli gene occur more frequently in the I type than in the G type, while microsatellite instability and alterations in the $p 73$ gene are more common in the $G$ type than in the I type. Microsatellite instability in the G type is usually associated with inactivation of hMLH1 following the promoter hypermethylation. The caudal-related homeobox gene 2 (CDX2) acts as an intestine-specific transcription factor and is expressed in I type GCs at high levels. CDX2 upregulates the expression of goblet-specific MUC2 [4].

Cytokeratin (CK), an intermediate filament observed mainly in epithelial cells, is an important cytoskeletal component involved in fixation of the nucleus and maintenance of cell morphology. CK consists of 20 subtypes, whose expression depends primarily on the epithelial cell type and the degree of differentiation [5]. The expression of CK8, CK18 and CK19 is observed at all levels of gastric mucosa, but CK20 expression is limited to mature superficial foveolar epithelium [6-8]. CK7 expression is absent in normal gastric mucosa but is observed in chronic mucosal irritation conditions such as $H$. pylori gastritis in the basal localization [9]. There are many reports of CK expression in various organs and the findings suggest that the CK expression profiles of metastatic cancers correspond to those of the primary sites [10-12]. In particular, in the gastrointestinal tract, colorectal carcinomas demonstrate a CK7-/CK20+ expression pattern, whereas adenocarcinomas of foregut origin demonstrate a $\mathrm{CK} 7+1$ CK20 - expression pattern [10-13]. Profiles for CK expression patterns may be helpful; however, the total number of GCs in each previous report about the expression of CK subtypes is one hundred cases at most $[5,6,9,12$ 21].

The aim of the present study was to analyze the CK expression patterns in a large number of GCs and to investigate how the $C K$ expression patterns correlate with clinicopathologic parameters, histology, mucin phenotypes or several tumor-related molecules. Because the functional and biological properties of the GCs may re- flect the tumors' ability to produce certain CKs, it would be of interest to determine which factors are best correlated with the CK immunophenotype.

\section{Materials and Methods}

Tissue Samples and Tissue Microarray Construction

The surgical pathology files of the Hiroshima University Hospital and its affiliated hospitals were used to randomly select 870 GCs from 51 adenocarcinomas of the esophagogastric junction (AEG) and 819 distal GCs (corpus or antrum). Surgically resected specimens were routinely fixed in $10 \%$ buffered formalin and examined macroscopically. Tumor staging was performed according to the Union Internationale Contre le Cancer (UICC) system [22]. There were 403 T1, 257 T2, 184 T3 and 26 T4 in these 870 cases. Nodal metastasis was present in 366 patients (42\%). Tumor staging revealed 500 stage I, 152 stage II, 154 stage III and 64 stage IV. GCs were histologically classified as 507 intestinal type and 363 diffuse type according to the Lauren classification system. The 507 intestinal type GCs included 63 papillary, 173 well-differentiated tubular and 271 moderately differentiated tubular adenocarcinomas. The 363 diffuse type GCs consisted of 297 poorly differentiated adenocarcinomas, 52 signet-ring cell carcinomas and 14 mucinous adenocarcinomas according to the WHO histological classification. In addition, the GCs were classified as 63 papillary (pap), 173 well differentiated tubular (tub1), 271 moderately differentiated tubular (tub2), 75 solid type poorly differentiated (por1), 222 non-solid type poorly differentiated (por2) adenocarcinomas and 52 signet-ring cell carcinoma (sig) and 14 mucinous adenocarcinomas (muc) according to the Japanese Classification of Gastric Carcinoma [23]. In accordance with the Ethical Guidelines for Human Genome/ Gene Research enacted by the Japanese Government, tissue specimens were collected and used after approval from the Ethical Review Committee of the Hiroshima University School of Medicine and from the ethical review committees of collaborating organizations.

The two most representative portions to be sampled for the tissue microarrays (TMAs) were carefully selected from different intratumoral areas in each case and marked on the HE-stained slide. Two superficial areas in mucosal GCs, and one superficial area and one deep area in GCs that had invaded beyond the submucosa were selected. A 2-mm-diameter tissue core of each donor block was punched out and transferred to a recipient block with a maximum of 48 cores using a tissue microarrayer (AZUMAYA KIN-1, Tokyo, Japan). 5- $\mu$ m-thick sections were cut from the recipient block and transferred to glass slides. HE staining was performed on TMA for confirmation of the tumor tissue. Each tissue-array block contained 21 cases of GC and four cases of nonneoplastic stomach samples.

\section{Immunohistochemistry}

A Dako Envision Kit (Dako, Carpinteria, Calif., USA) was used for immunohistochemical analysis of all markers. In brief, sections were pretreated by microwaving $(500 \mathrm{~W})$ in a citrate buffer ( $\mathrm{pH}$ 6.0) for $15 \mathrm{~min}$ to retrieve antigenicity. After endogenous peroxidase activity was blocked with $3 \% \mathrm{H}_{2} \mathrm{O}_{2}$-methanol for 10 min, sections were incubated with normal goat serum (Dako) for 
Table 1. Antibodies used in the current study and their positive rates in 870 GCs

\begin{tabular}{lllll}
\hline Antibody & Clone & Dilution & Source & $\begin{array}{l}\text { Positive cases } \\
\text { n (\%) }\end{array}$ \\
\hline CK7 & OV-TL 12/30 & $1: 50$ & DAKO, Carpinteria, Calif., USA & $648(74)$ \\
CK20 & IT-Ks 20.8 & $1: 50$ & DAKO, Carpinteria, Calif., USA & $232(27)$ \\
MUC5AC & CLH2 & $1: 50$ & Novocastra, Newcastle, UK & $519(60)$ \\
MUC6 & CLH5 & $1: 50$ & Novocastra, Newcastle, UK & $233(27)$ \\
MUC2 & Ccp58 & $1: 50$ & Novocastra, Newcastle, UK & $199(23)$ \\
CD10 & 56C6 & $1: 50$ & Novocastra, Newcastle, UK & $71(8)$ \\
p53 & DO-7 & $1: 50$ & Novocastra, Newcastle, UK & $293(34)$ \\
EGFR & EGFR.113 & $1: 50$ & Novocastra, Newcastle, UK & $109(13)$ \\
CDX2 & AMT28 & $1: 20$ & BioGenex, San Ramon, Calif., USA & $183(21)$ \\
$\beta$-Catenin & $14 / \beta-c a t e n i n$ & $1: 50$ & BD Biosciences, San Jose, Calif., USA & $140(16)$ \\
\hline
\end{tabular}

The cutoff point for antibody reactivity necessary to define a result as positive was staining of at least $10 \%$ of cancer cells in the TMAs.

\begin{abstract}
20 min to block nonspecific antibody binding sites. Sections were then incubated with the following primary antibodies: anti-CK7, anti-CK20, anti-MUC5AC, anti-MUC6, anti-MUC2, anti-CD10, anti-p53, anti-EGFR, anti-CDX2 and anti- $\beta$-catenin. Suppliers and working dilutions are noted in table 1 . Sections were incubated with a primary antibody for $1 \mathrm{~h}$ at $25^{\circ} \mathrm{C}$, followed by incubations with peroxidase-labeled anti-rabbit or mouse IgG for 60 min. Staining was completed with a $10-$ min incubation with the substrate-chromogen solution. The sections were counterstained with $0.1 \%$ hematoxylin. Appropriate positive and negative control samples were used.
\end{abstract}

\section{Evaluation of Positive Cases and Cutoff-Point Thresholds}

Immunostaining results were evaluated independently by 3 investigators (H.T., K.S. and M.M.) and when the evaluations differed, a decision was made by consensus while the investigators reviewed the specimen with a multihead microscope. Neoplastic tissue was evaluated semiquantitatively at magnifications of $\times 100$ and $\times 400$. Cytoplasmic immunoreactivity for CK7, CK20, MUC5AC, MUC6 and MUC2, membranous reactivity for CD10 and EGFR, and nuclear reactivity for p53, CDX 2 and $\beta$-catenin were assessed (fig. 1). The cutoff point for antibody reactivity necessary to define a result as positive was staining of more than $10 \%$ tumor cells in the TMAs.

\section{CK Expression Profiles and Mucin Phenotypes}

The 870 GCs were evaluated according to the CK7 and CK20 staining pattern and classified into four main groups: (1) coexpression of CK7 and CK20 (CK7+/CK20+), (2) no expression of CK7 and CK20 (CK7-/CK20-), (3) only CK7 expression (CK7+/ CK20-) and (4) only CK20 expression (CK7-/CK20+). The criteria [24] for classification of $G$ type and I type were as follows: GCs in which more than $10 \%$ of the cells displayed the gastric (MUC5AC and/or MUC6) or intestinal epithelial cell phenotype (MUC2 and/or CD10) were G type or I type, respectively. Those sections that showed both $G$ and I types were classified as GI type, and those that lacked both $\mathrm{G}$ and I types were classified as $\mathrm{N}$ type.
Statistical Methods

Associations between CK expression profiling and clinicopathologic variables, or immunostaining for various markers were analyzed by the $\chi^{2}$ test. $\mathrm{p}<0.001$ was considered statistically significant.

\section{Results}

CK Expression Patterns in GCs and Their Correlation with Clinicopathologic Parameters

Immunohistochemical results in the current study are shown in table 1 . The 870 GCs included 648 (74\%) cases with CK7 expression and 232 (27\%) cases with CK20 expression, and were classified into 156 (17\%) cases with CK7+/CK20+ pattern, 492 (57\%) cases with CK7+/CK20pattern, 76 (9\%) cases with CK7-/CK20+ pattern and 146 (17\%) cases with CK7-/CK20- pattern. We investigated the relation between $\mathrm{CK}$ expression patterns and the clinicopathologic parameters including age, sex, tumor location, $\mathrm{T}$ grade, $\mathrm{N}$ grade, $\mathrm{M}$ grade, staging and histological type according to the Lauren classification. As shown in table 2, the CK7-/CK20- pattern was observed more frequently in the diffuse type of GC than in the intestinal type $(p=0.0003)$. In contrast, no differential trend was found between other CK expression patterns and clinicopathologic parameters. Regarding the Japanese Classification of Gastric Carcinomas, the CK7-/CK20 - pattern was observed more frequently in the undifferentiated type (por1, por2, sig and muc) than in the differentiated type GC (pap, tub1 and tub2) (fig. 2; p = 0.0003). Furthermore, the analysis according to the WHO classification also yielded a similar result. 

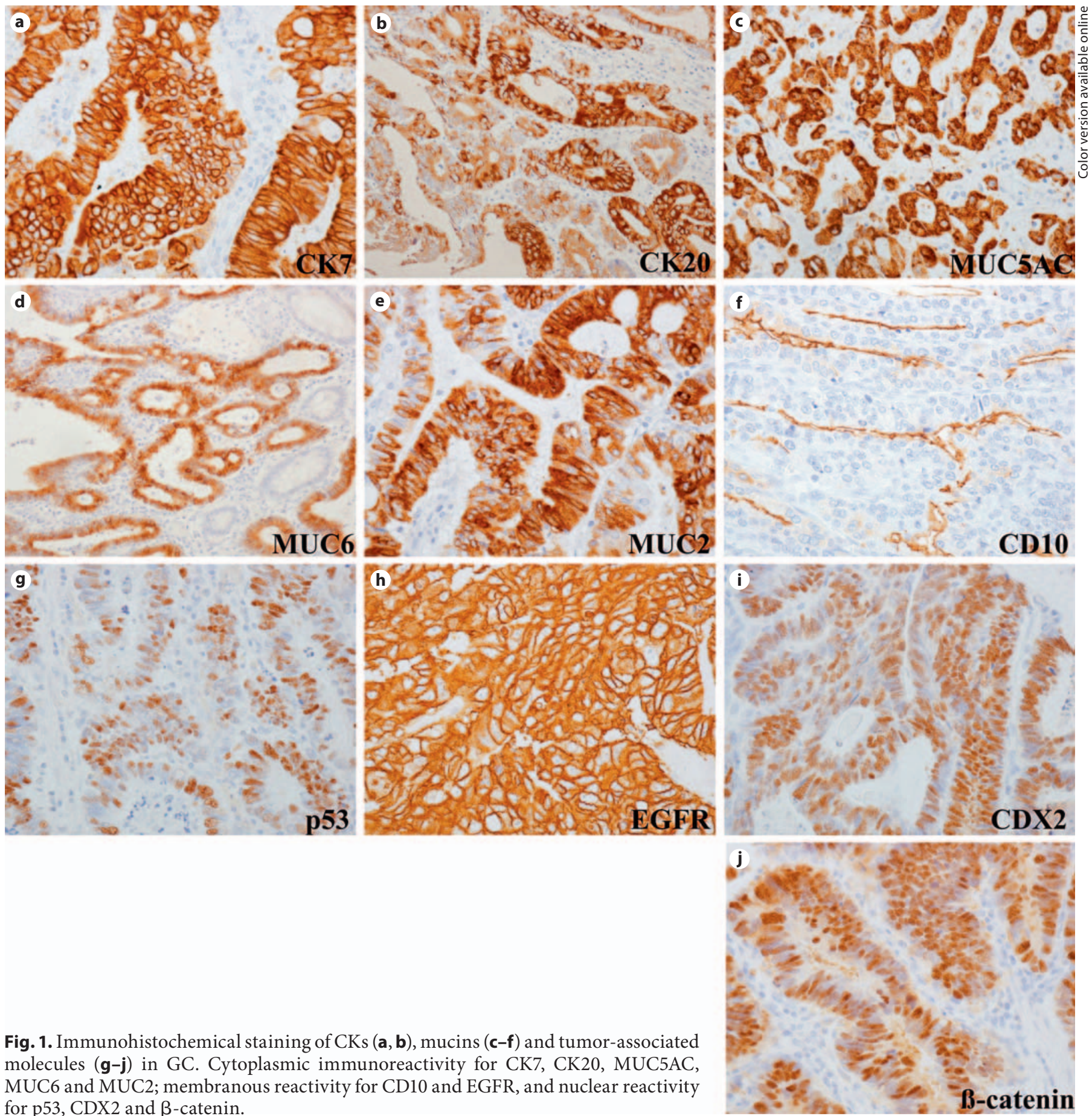

Fig. 1. Immunohistochemical staining of CKs (a, b), mucins (c-f) and tumor-associated molecules $(\mathbf{g}-\mathbf{j})$ in GC. Cytoplasmic immunoreactivity for CK7, CK20, MUC5AC, MUC6 and MUC2; membranous reactivity for CD10 and EGFR, and nuclear reactivity for $\mathrm{p} 53, \mathrm{CDX} 2$ and $\beta$-catenin.

sion, 233 (27\%) cases with MUC6 expression, 199 (23\%) cases with MUC2 expression and $71(8 \%)$ cases with CD10 expression. They were classified into 313 (36\%) G type, 196 (23\%) GI type, 149 (17\%) I type and 212 (24\%) $\mathrm{N}$ type. The distribution of each mucin phenotype and 
Table 2. Relationships between CK expression patterns and clinicopathological findings in 870 GCs

\begin{tabular}{|c|c|c|c|c|c|c|}
\hline CK pattern & & $\begin{array}{l}\text { CK7+/CK20+ } \\
(\mathrm{n}=156)\end{array}$ & $\begin{array}{l}\text { CK7+/CK20- } \\
(\mathrm{n}=492)\end{array}$ & $\begin{array}{l}\text { CK7-/CK20+ } \\
(\mathrm{n}=76)\end{array}$ & $\begin{array}{l}\text { CK7-/CK20- } \\
(\mathrm{n}=146)\end{array}$ & $\mathrm{p}$ value \\
\hline \multirow[t]{2}{*}{ Age } & $>65$ years & $74(47)$ & $258(52)$ & $35(46)$ & $79(54)$ & \multirow[t]{2}{*}{ NS } \\
\hline & $\leq 65$ years & $82(53)$ & $234(48)$ & $41(54)$ & $67(46)$ & \\
\hline \multirow[t]{2}{*}{ Sex } & Male & $93(60)$ & $322(65)$ & $41(54)$ & $97(66)$ & \multirow[t]{2}{*}{ NS } \\
\hline & Female & $63(40)$ & $170(35)$ & $35(46)$ & $49(34)$ & \\
\hline \multirow{2}{*}{ Tumor location } & AEG & $10(6)$ & $28(6)$ & $6(8)$ & $7(5)$ & \multirow[t]{2}{*}{ NS } \\
\hline & Distal GC & $146(94)$ & $464(94)$ & $70(92)$ & $139(95)$ & \\
\hline \multirow[t]{2}{*}{ T grade } & $\mathrm{T} 1$ & $91(58)$ & $214(43)$ & $29(38)$ & $69(47)$ & \multirow[t]{2}{*}{ NS } \\
\hline & T2/T3/T4 & $65(42)$ & $278(57)$ & $47(62)$ & $77(53)$ & \\
\hline \multirow[t]{2}{*}{$\mathrm{N}$ grade } & No & $107(69)$ & $279(57)$ & $36(47)$ & $82(56)$ & \multirow[t]{2}{*}{ NS } \\
\hline & N1 & $49(31)$ & $213(43)$ & $40(53)$ & $64(44)$ & \\
\hline \multirow[t]{2}{*}{$M$ grade } & M0 & 155 (99) & $484(98)$ & $76(100)$ & $146(100)$ & \multirow[t]{2}{*}{ NS } \\
\hline & M1 & $1(1)$ & $8(2)$ & 0 & 0 & \\
\hline \multirow[t]{2}{*}{ Staging } & Stage I & $106(68)$ & $277(56)$ & $36(47)$ & $81(55)$ & \multirow[t]{2}{*}{ NS } \\
\hline & Stage II/III/IV & $50(32)$ & $215(44)$ & $40(53)$ & $65(45)$ & \\
\hline \multirow[t]{2}{*}{ Histology } & Intestinal type & $105(67)$ & $285(58)$ & $52(68)$ & $61(42)$ & \multirow[t]{2}{*}{0.0003} \\
\hline & Diffuse type & $51(33)$ & $207(42)$ & $24(32)$ & $85(58)$ & \\
\hline
\end{tabular}

Values in parentheses are percentages. AEG = Adenocarcinoma of the esophagogastric junction; $\mathrm{NS}=$ not significant. A $\mathrm{p}<0.001$ was considered statistically significant by $\chi^{2}$ test. Tumor staging was classified according to the criteria of the International Union Against Cancer TNM classification of malignant tumors. Histology was according to the Lauren classification system.

histological type according to the Japanese Classification of Gastric Carcinomas is shown in figure 2. The $\mathrm{N}$ type was observed more frequently in the undifferentiated type GC.

\section{Association of Expression between CK Patterns and \\ Various Molecules}

We then investigated the association between CK expression patterns and various molecules in the GCs. Of the 870 GCs examined, each tumor-associated molecule was detected in 293 (34\%) cases for p53, 109 (13\%) cases for EGFR, 183 (21\%) cases for CDX2 and 140 (16\%) cases for $\beta$-catenin (table 1 ). There are statistically significant associations between CK7 and MUC5AC expression, CK7 and MUC6 expression, CK20 and MUC2 expression, and CK20 and CDX2 (p<0.0001) (fig. 3).

\section{Association of CK Expression Patterns with Mucin \\ Phenotypes}

The relationship between each CK expression pattern and mucin phenotype in the 870 GCs was analyzed. As shown in figure 4 , there are statistically significant associations between CK7 expression without CK20 expres- sion and G type, CK7 expression and GI type, CK20 expression without CK7 expression and I type, and neither of them and $\mathrm{N}$ type, respectively $(\mathrm{p}<0.0001)$.

\section{Discussion}

Much interest has focused on CK immunoprofiles in the classification of carcinomas, in particular the CK7 and CK20 profiles. There are many reports on the expression of CK7 and CK20 in relatively small numbers of GC cases [5, 6, 9, 12-21]. CK7 immunoreactivity is reported to range from 10 to $75 \%$, while CK20 expression is frequently reported to range from 30 to $50 \%$. Our results are also consistent with the findings of previous reports. The differences of positive rates in these previous reports are possibly due to evaluation scales and case groups. In this study, we used the TMA method to examine each molecule expression in the GCs. Although minute TMAs cannot ensure representative areas of donor specimen, we used 2-mm-diameter needles, which are large enough to evaluate the morphological appearance if representative regions are carefully selected with HE slides $[25,26]$. In 


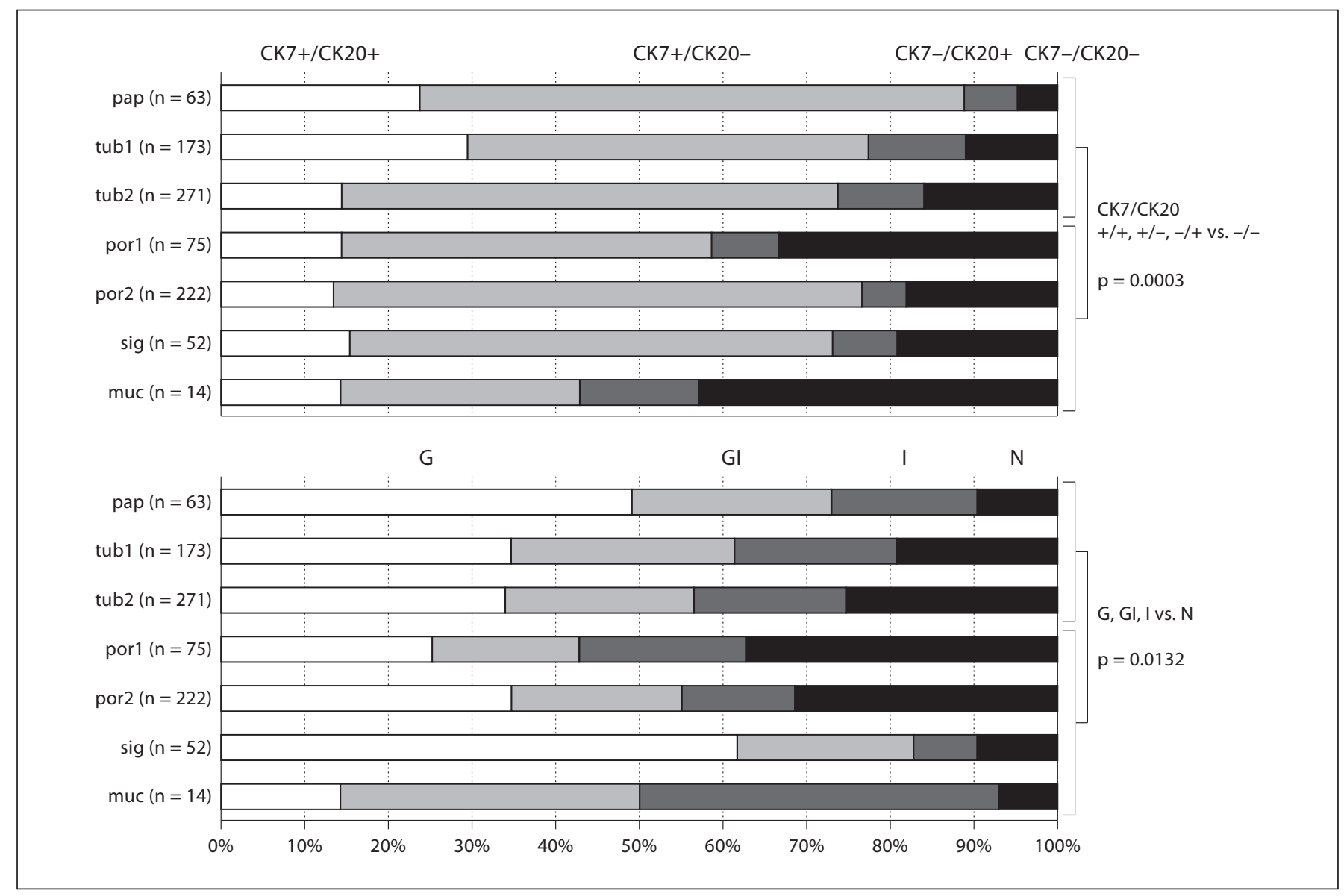

Fig. 2. The relationships between detailed histological type and CK expression patterns and mucin phenotypes in $870 \mathrm{GCs}$. The 870 GCs were histologically classified as 507 of the differentiated type (papillary adenocarcinoma or tubular adenocarcinoma) and 363 of the undifferentiated type (poorly differentiated

terms of the possible diversity of histological components or molecular abnormality in the GCs, several previous reports have shown an excellent concordance between the results obtained from TMAs and those from full sections [27, 28]. Furthermore, the effects of intratumoral heterogeneity can be averaged out in such a large-scale analysis as the present study. It is unlikely that the use of TMA biased the outcome.

To clarify the significance of the CK expression patterns, we analyzed the relation between $\mathrm{CK}$ expression patterns and the clinicopathologic parameters, histology, mucin phenotype or several tumor-related molecules. Histologically, expression of CK7 and/or CK20 showed a tendency toward a high positive rate in differentiated type GC and a low positive rate in undifferentiated type GC. adenocarcinoma, signet-ring cell carcinoma or mucinous adenocarcinoma). There are statistically significant associations between the undifferentiated type and neither CK7 nor CK20 expression, and the undifferentiated type and $\mathrm{N}$ mucin phenotype.
This may reflect a loss of the ability to produce the CKs along with a decrease in histological differentiation in neoplastic cells. However, CK7 and/or CK20 expression was not associated with any other clinicopathologic features, consistent with the previous report [17, 29]. GCs have been classified into four mucin phenotypes. Previous reports provided evidence that mucin expression is closely associated with the differentiation of GCs [30, 31]. In the present study, G type was correlated with CK7 expression, especially in the absence of CK20 expression, whereas I type was correlated with CK20 expression, especially in the absence of CK7 expression. This result demonstrated statistical significance, but neither CK7 nor CK20 was sufficient for the discrimination of the mucin phenotype due to low sensitivity and specificity. In the present study, 


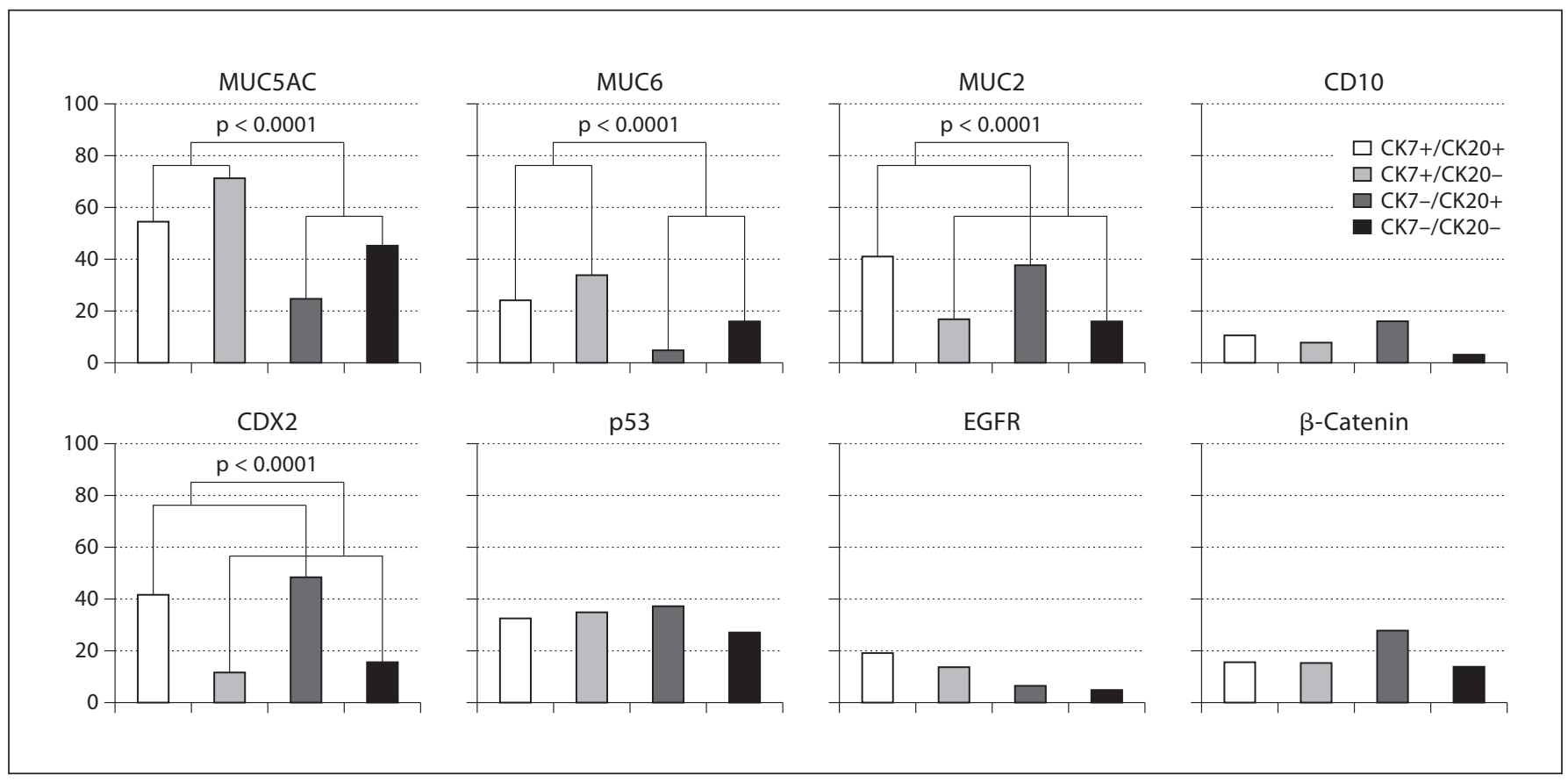

Fig. 3. The relationships between CK expression patterns and various markers in 870 GCs. There are statistically significant associations between CK7 and MUC5AC expression, CK7 and MUC6 expression, CK20 and MUC2 expression, and CK20 and CDX2 (p < $0.0001)$.

Fig. 4. The relationships between each CK expression pattern and mucin phenotypes in 870 GCs. There are statistically significant associations between CK7 expression without CK20 expression and G mucin phenotype, CK7 expression and GI mucin phenotype, CK20 expression without CK7 expression and I mucin phenotype, and neither of them and $\mathrm{N}$ mucin phenotype $(\mathrm{p}<0.0001)$.

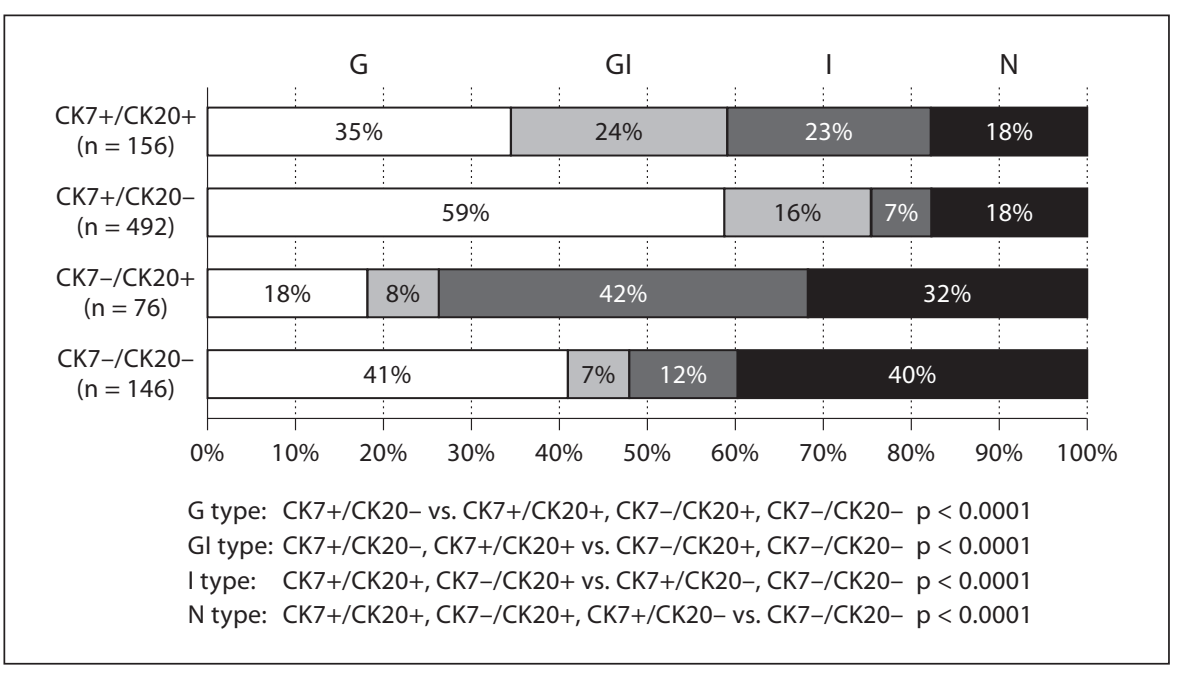

the positive expression of CK20 was frequently observed in GCs with the I type and showed significant correlation with the positive expression of $\mathrm{Cdx} 2$. There is no previous report showing a direct association between CK20 and Cdx2. Chan et al. [32] reported that CK20 is directly regulated by Cdx1. Therefore, there may also be a close correlation between CK20 and CDX2.
In summary, GCs showed heterogenous CK expression representing their histological features. Therefore, a single CK and its combination does not always provide diagnostic value in differentiating GCs. Advances in our understanding of the genetic and molecular bases of GC according to each CK expression, however, may lead to new therapy. 


\section{Acknowledgements}

We thank Ms. Emiko Hisamoto and Mr. Shinichi Norimura for their excellent technical assistance and advice. This work was carried out with the kind cooperation of the Research Center for Molecular Medicine, Faculty of Medicine, Hiroshima University. We also thank the Analysis Center of Life Science, Hiro- shima University, for the use of its facilities. This work was supported in part by Grants-in-Aid for Cancer Research from the Ministry of Education, Culture, Science, Sports and Technology of Japan and in part by a Grant-in-Aid for the Third Comprehensive 10-Year Strategy for Cancer Control and for Cancer Research from the Ministry of Health, Labour and Welfare of Japan.

\section{References}

1 Carneiro F, Sobrinho-Simoes M: Metastatic pattern of gastric carcinoma. Hum Pathol 1996;27:213-214

2 Yasui W, Oue N, Kitadai Y, Nakayama H: Recent advances in molecular pathobiology of gastric carcinoma; in Kaminishi M, Takubo K, Mafune K (eds): The Diversity of Gastric Carcinoma: Pathogenesis, Diagnosis and Therapy. Tokyo, Springer, 2005, pp 51-71.

$\checkmark 3$ Tatematsu M, Tsukamoto T, Inada K: Stem cells and gastric cancer: role of gastric and intestinal mixed intestinal metaplasia. Cancer Sci 2003;94:135-141.

4 Yasui W, Sentani K, Motoshita J, Nakayama $\mathrm{H}$ : Molecular pathobiology of gastric cancer. Scand J Surg 2006;95:225-231.

5 Chu PG, Weiss LM: Keratin expression in human tissues and neoplasms. Histopathology 2002;40:403-439.

6 Chu P, Wu E, Weiss LM: Cytokeratin 7 and cytokeratin 20 expression in epithelial neoplasms: a survey of 435 cases. Mod Pathol 2000;13:962-972.

$\checkmark 7$ Moll R: Cytokeratins in the histological diagnosis of malignant tumors. Int $\mathrm{J}$ Biol Markers 1994;9:63-69.

$>8$ Moll R, Lowe A, Laufer J, Franke WW: Cytokeratin 20 in human carcinomas. A new histodiagnostic marker detected by monoclonal antibodies. Am J Pathol 1992;140:427-447.

$\checkmark 9$ Schwerer MJ, Baczako K: Expression of cytokeratins typical for ductal and squamous differentiation in the human stomach: an immunohistochemical study of normal foveolar epithelium, Helicobacter pylori gastritis and intestinal metaplasia. Histopathology 1996;29:131-137.

-10 Ramaekers F, van Niekerk C, Poels L, Schaafsma E, Huijsmans A, Robben H, Schaart G, Vooijs P: Use of monoclonal antibodies to keratin 7 in the differential diagnosis of adenocarcinomas. Am J Pathol 1990; 136:641-655.

-11 Tot T: Cytokeratins 20 and 7 as biomarkers: usefulness in discriminating primary from metastatic adenocarcinoma. Eur J Cancer 2002;38:758-763.

-12 Park SY, Kim HS, Hong EK, Kim WH: Expression of cytokeratins 7 and 20 in primary carcinomas of the stomach and colorectum and their value in the differential diagnosis of metastatic carcinomas to the ovary. Hum Pathol 2002;33:1078-1085.
13 Moll R: Cytokeratins as markers of differentiation in the diagnosis of epithelial tumors. Subcell Biochem 1998;31:205-262.

14 Wauters CC, Smedts F, Gerrits LG, Bosman FT, Ramaekers FC: Keratins 7 and 20 as diagnostic markers of carcinomas metastatic to the ovary. Hum Pathol 1995;26:852-855.

15 Kirchner T, Muller S, Hattori T, Mukaisyo K, Papadopoulos T, Brabletz T, Jung A: Metaplasia, intraepithelial neoplasia and early cancer of the stomach are related to dedifferentiated epithelial cells defined by cytokeratin-7 expression in gastritis. Virchows Arch 2001;439:512-522.

16 Ormsby AH, Goldblum JR, Rice TW, Richter JE, Gramlich TL: The utility of cytokeratin subsets in distinguishing Barrett's-related oesophageal adenocarcinoma from gastric adenocarcinoma. Histopathology 2001;38: 307-311.

17 Kim MA, Lee HS, Yang HK, Kim WH: Cytokeratin expression profile in gastric carcinomas. Hum Pathol 2004;35:576-581.

18 Shen B, Ormsby AH, Shen C, Dumot JA, Shao YW, Bevins CL, Gramlich TL: Cytokeratin expression patterns in noncardia, intestinal metaplasia-associated gastric adenocarcinoma: implication for the evaluation of intestinal metaplasia and tumors at the esophagogastric junction. Cancer 2002;94: $820-831$.

19 Gurbuz Y, Kose N: Cytokeratin expression patterns of gastric carcinomas according to Lauren and Goseki classification. Appl Immunohistochem Mol Morphol 2006;14:303308.

20 Lee MJ, Lee HS, Kim WH, Choi Y, Yang M: Expression of mucins and cytokeratins in primary carcinomas of the digestive system. Mod Pathol 2003; 16:403-410.

21 Xue L, Zhang X, Li Y, Yang H, Li X, Mi J, Wang H, Wang J, Yan X: Differences of immunophenotypic markers and signaling molecules between adenocarcinomas of gastric cardia and distal stomach. Hum Pathol 2011;42:594-601.

22 Sobin LH, Wittekind CH (eds): TNM Classification Of Malignant Tumors, ed 6. New York, Wiley, 2002, pp 65-68.

23 Japanese Gastric Cancer Association: Japanese classification of gastric carcinoma, 3rd English edition. Gastric Cancer 2011;14:101112 .
24 Mizoshita T, Tsukamoto T, Nakanishi H, Inada K, Ogasawara N, Joh T, Itoh M, Yamamura Y, Tatematsu M: Expression of Cdx2 and the phenotype of advanced gastric cancers: relationship with prognosis. J Cancer Res Clin Oncol 2003;129:727-734.

25 Sauter G, Mirlacher M: Tissue microarrays for predictive molecular pathology. J Clin Pathol 2002;55:575-576.

26 Sentani K, Oue N, Tashiro T, Sakamoto N, Nishisaka T, Fukuhara T, Taniyama K, Matsuura H, Arihiro K, Ochiai A, Yasui W: Immunohistochemical staining of reg IV and claudin-18 is useful in the diagnosis of gastrointestinal signet ring cell carcinoma. Am J Surg Pathol 2008;32:1182-1189.

27 Hoos A, Urist MJ, Stojadinovic A, Mastorides S, Dudas ME, Leung DH, Kuo D, Brennan MF, Lewis JJ, Cordon-Cardo C: Validation of tissue microarrays for immunohistochemical profiling of cancer specimens using the example of human fibroblastic tumors. Am J Pathol 2001;158:1245-1151.

28 Lee HS, Cho SB, Lee HE, Kim MA, Kim JH, Park do J, Kim JH, Yang HK, Lee BL, Kim WH: Protein expression profiling and molecular classification of gastric cancer by the tissue array method. Clin Cancer Res 2007; 13:4154-4163.

29 Gulmann C, Counihan I, Grace A, Patchett S, Leen E, Leader M, Kay E: Cytokeratin 7/20 and mucin expression patterns in oesophageal, cardia and distal gastric adenocarcinomas. Histopathology 2003;43:453-461.

30 Pinto-de-Sousa J, David L, Reis CA, Gomes R, Silva L, Pimenta A: Mucins MUC1, MUC2, MUC5AC and MUC6 expression in the evaluation of differentiation and clinicobiological behaviour of gastric carcinoma. Virchows Arch 2002;440:304-310.

31 Pinto-de-Sousa J, Reis CA, David L, Pimenta A, Cardoso-de-Oliveira M: MUC5B expression in gastric carcinoma: relationship with clinico-pathological parameters and with expression of mucins MUC1, MUC2, MUC5AC and MUC6. Virchows Arch 2004;444: 224-230.

32 Chan CW, Wong NA, Liu Y, Bicknell D, Turley H, Hollins L, Miller CJ, Wilding JL, Bodmer WF: Gastrointestinal differentiation marker cytokeratin 20 is regulated by homeobox gene CDX1. Proc Natl Acad Sci USA 2009;106:1936-1941. 